\title{
CHEMISCHE ABHÄNGIGKEIT DURCH MARIHUANAKONSUM: VOM VERGNÜGEN ZUR KRANKHEIT. EIN ERNSTES PROBLEM IM GESUNDHEITSWESEN IN BRASILIEN
}

\section{ORIGINALER ARTIKEL}

DIAS, Amanda de Araújoํㅜㄹ, DIAS, Édina Lúcia de Araújo², OLIVEIRA, Ciane Martins de $^{3}$, DENDASCK, Carla Viana ${ }^{4}$, OLIVEIRA, Euzébio de ${ }^{5}$

DIAS, Amanda de Araújo Dias. Et al. Chemische Abhängigkeit durch Marihuanakonsum: vom Vergnügen zur Krankheit. Ein ernstes Problem im Gesundheitswesen in Brasilien. Revista Científica Multidisciplinar Núcleo do Conhecimento. Jahrgang 06, Ed. 03, Vol. 11, S. 78-86. März 2021. ISSN: 24480959,

Zugangslink: https://www.nucleodoconhecimento.com.br/gesundheit/marihuanakons um, DOI: 10.32749/nucleodoconhecimento.com.br/gesundheit/marihuanakonsum

\section{ABSTRAKT}

Einleitung: Der Marihuanakonsum hat weltweit zugenommen und ist damit die am weitesten verbreitete illegale Droge der Welt. Schätzungen zufolge konsumierten zwischen 167 und 315 Millionen Menschen zwischen 15 und 64 Jahren in den letzten zehn Jahren illegale Drogen. In Brasilien nutzen rund 7,5 Millionen Studenten, verteilt in rund 2.400 Einrichtungen, das Kraut. Methode: Eine beschreibende Studie wurde auf der Grundlage einer Literaturliteratur-Rezension durchgeführt. Die Forschung wurde über die Scielo-Datenbank und die virtuelle Gesundheitsbibliothek (BVS)

\footnotetext{
${ }^{1}$ Medizinischer Akademiker am Universitätszentrum des Staates Pará - CESUPA.

2 Psychologe. Postgraduate in Mental Health bei Escola Superior da Amazônia - ESAMAZ.

${ }^{3}$ PhD in Medizinischer Genetik. Professor und Forscher am Universitätszentrum des Staates Pará (CESUPA).

${ }^{4}$ Theologe, PhD in Clinical Psychoanalysis. Er arbeitet seit 15 Jahren mit Scientific Methodology (Research Method) in der wissenschaftlichen Produktionsberatung von Master- und Doktoranden. Der Spezialist für Marktforschung und -forschung konzentrierte sich auf Gesundheit.

${ }^{5} \mathrm{PhD}$ in Medizin/Tropische Krankheiten. Professor und Forscher an der Föderalen Universität Pará - UFPA.
}

RC: 80477

Disponível em: 
durchgeführt. Ergebnisse UND Diskussion: In Bezug auf die Auswirkungen von Marihuana, in einigen Studien Symptome im Zusammenhang mit Freizeitgebrauch und Missbrauch dieser Droge berichtet. Auf akademischer Ebene können die Auswirkungen des Marihuanakonsums jedoch in akute und chronische Symptome unterteilt werden. Akute Effekte werden als Euphorie, körperliche Effekte und psychische Effekte wie Depression, Halluzination, Illusion, Schläfrigkeit und Beeinträchtigungen der Konzentration und Desfristgedächtnis klassifiziert. Fazit: Es gibt Hinweise, die erklären können, warum Einzelpersonen sich für den Konsum von Marihuana entscheiden. Meistens sind die akuten Auswirkungen von Euphorie, Vergnügen und Entspannung gewünscht, im Gegensatz dazu wird beobachtet, dass die Schäden im Zusammenhang mit diesem Gebrauch am Ende die illusorischen Vorteile, die das Medikament bietet, zu überwinden, so dass Freude zu einer Krankheit wird.

Stichworte: Marihuana, Konsum, Genuss, Krankheit.

\section{EINLEITUNG}

Die Gewohnheit des Drogenkonsums ist seit der Antike in Gesellschaften präsent und etabliert, und die erste Aufzeichnung des Marihuana-Konsums (Cannabis) stammt aus mindestens zehntausend Jahren, in denen es Aufzeichnungen über den Anbau und Gebrauch verschiedener Zivilisationen auf der ganzen Welt gibt, da sie die älteste in Asien und im Nahen Osten sind und später in Afrika, Amerika und anderen Regionen ankommen (ROSA; ROSA, 2018).

Schätzungen zufolge konsumierten zwischen 167 und 315 Millionen Menschen zwischen 15 und 64 Jahren in den letzten zehn Jahren illegale Drogen, was 3,6 bis $6,9 \%$ der Weltbevölkerung entspricht. In diesem Zusammenhang hat der Marihuanakonsum weltweit zugenommen und ist damit die am weitesten verbreitete illegale Droge der Welt. Marihuana wird heute schätzungsweise von 125 bis 203

RC: 80477

Disponível em: 
Millionen Menschen konsumiert, mit der höchsten Prävalenz in Zentral- und Westafrika (GARCIA, 2014).

In Brasilien, etwa 7,5 Millionen Universitätsstudenten, verteilt in etwa 2.400 Institutionen verwenden das Kraut, und die Verwendung der Substanz ist eine häufige Praxis, viel diskutiert von den Laienmedien und analysiert durch einige wissenschaftliche Studien (GARCIA, 2014; FERNANDES et al., 2017).

Da der Konsum von Marihuana in unserem sozialen Umfeld immer häufiger vorkommt, ist es äußerst wichtig, dass Studien durchgeführt werden, um das Wissen über seine chemischen, psychologischen und sozialen Auswirkungen aufgrund der Bedeutung, die sie darstellen, zu erweitern.

Darüber hinaus gab es in den letzten Jahren Diskussionen über die Vor- und Nachteile im Zusammenhang mit der Legalisierung dieser Droge, und für Profis und die Bevölkerung in der Lage, ihre Meinung zu geben, ist es notwendig, zuerst zu verstehen, wie Marihuana den Körper kurz- und langfristig verändert (COUTINHO; ARAÚJO; GONTIÈS, 2004).

In diesem Zusammenhang wird diese Arbeit durch die Erstellung gerechtfertigt, um das vorhandene Wissen über den Konsum von Marihuana zu verfeinern und die akademische Gesellschaft über den Prozess der Freude und Krankheit, mit dem diese Droge direkt verbunden ist, zu aktualisieren.

Diese Überprüfung zielt darauf ab, die wichtigsten Aspekte der chemischen Abhängigkeit durch Marihuana-Konsum zu adressieren, sowie Konzepte über die angenehmen Empfindungen der Verwendung dieses Medikaments und wie es zu dem Wunsch beiträgt, seine Verwendung fortzusetzen. Es ist auch von Interesse für diesen Artikel, die physischen und psychischen Folgen zu diskutieren, die Marihuana im Körper entsteht und einen Krankheitsprozess durch seinen längeren Gebrauch

RC: 80477

Disponível em:

https://www.nucleodoconhecimento.com.br/gesundheit/marihuanakonsum 
charakterisiert und $\mathrm{zu}$ besseren Informationen über die Gründe für die Nichtverwendung dieses Medikaments beiträgt.

\section{MATERIAL UND METHODE}

Die in dieser Studie verwendete Methodik war eine beschreibende Studie, die auf einer Literaturrecherche beruhte. Die bibliographische Forschung wurde über die Scielo-Datenbank und die Virtual Health Library (BVS) durchgeführt. Um die Abstracts Portugiesisch in solchen Basen zu durchsuchen, wurden die folgenden Begriffe verwendet: Marihuana, Vergnügen und Krankheit. Nach diesem ersten Prozess wurden die Titel gelesen, und dann die Abstracts, um die Publikationen auszuwählen, die die Forschung interessierten, basierend auf den Anforderungen, die als Inklusions- und Ausschlusskriterien festgelegt wurden. Diese Forschung hatte als Aufnahmekriterien Artikel veröffentlicht im Zeitraum von 2002 bis 2020, in der portugiesischen Sprache, die die Themen im Zusammenhang mit Marihuana und seine wichtigsten physiologischen und pathologischen Auswirkungen auf den menschlichen Körper, in Bezug auf Körper und Geist. Ausschlusskriterien waren Papiere, die in einer Fremdsprache veröffentlicht wurden, die die Aufnahmekriterien nicht erfüllten. Schließlich wurden die ausgewählten Artikel analysiert und entstanden aus dem Schreiben der vorliegenden Arbeit.

\section{LITERATURREZENSION UND DISKUSSION}

\subsection{GRUNDBEGRIFFE IM ZUSAMMENHANG MIT MARIHUANA}

Marihuana ist ein Kraut, dessen wissenschaftlicher Name Cannabis ist. Im Lateinischen bedeutet Cannabis Hanf, den es die Pflanzenfamilie nennt, und es gibt drei Arten davon, Sativa, Indica und Ruderalis (FERRARI, 2016). Die Arten Sativa und Indica werden sowohl zum Rauchen als auch für verschiedene Anwendungen in der Küche und Medizin am häufigsten verwendet (CORTEZ, 2009).

RC: 80477

Disponível em:

https://www.nucleodoconhecimento.com.br/gesundheit/marihuanakonsum 
Seit Jahrhunderten verwendet die Bevölkerung Marihuanagras für medizinische Zwecke, zum Beispiel wiesen die Chinesen auf die medizinischen Eigenschaften von Marihuana als krampflössiv, beruhigend und schmerzstillend hin. Das Potenzial seiner medizinischen Eigenschaften bei der Behandlung von einigen Krankheiten ist bereits wissenschaftlich belegt, was weitere Studien erfordert, um seine Auswirkungen in diesem Bereich zu analysieren (RIBEIRO; MARQUES, 2008).

Cannabis Sativa enthält etwa 400 Chemikalien, mindestens 60 von ihnen Alkaloide, bekannt als Cannabinoide. Der wichtigste psychoaktive Bestandteil der Pflanze ist Tetrahydrocannabiol (THC), eine der Verbindungen des Krauts, die auch aus anderen Cannabinoiden wie Cannabidiol (CBD), Cannabinol (CBN) und Tetrahydrocanabivarin (THCV) besteht.Diese sind unterteilt in psychoaktive (Delta-8THC, Delta-9-THC und 11-Hydroxy-delta-9-THC) und nicht-psychoaktiv (Cannabidiol und Cannabinol). Delta-9-THC ist bekannt als die stärkste von Cannabinoiden (SOLOWIJ; PESA, 2008; CORTEZ, 2009).

\subsection{DIE WIRKUNG UND DAS VERGNÜGEN}

Der Hauptweg, Marihuana Gras zu verwenden, ist durch rauchen und die Bioverfügbarkeit von THC beim Rauchen beträgt etwa $20 \%$. Andere Formen der Verwendung können durch Haxixe durchgeführt werden, die auch geräuchert werden kann und höhere Konzentrationen von THC hat, und Hashoil oder Cannabis, das die flüssige und potenteste Form des intravenösen Medikaments ist. Beim Einatmen gelangt der durch das verbrannte Kraut erzeugte Rauch in die Lungenalveolos, dringt in die Durchblutung ein und erreicht das Gehirn in wenigen Minuten (CORTEZ, 2009).

Die oralen Absorptionsraten sind höher (90 bis 95\%) (30 das 45 Minuten) in Bezug auf die Lungenabsorption (50\%). Pharmakologische Wirkungen durch Lungenabsorption können zwischen 5 und 10 Minuten dauern (SOLOWIJ; PESA, 2008; CORTEZ, 2009).

RC: 80477

Disponível em:

https://www.nucleodoconhecimento.com.br/gesundheit/marihuanakonsum 
Die Durch das Medikament verursachten Spitzeneffekte treten in 30 Minuten auf und enden nach etwa zwei bis drei Stunden (CORTEZ, 2009).

Cannabinoide werden hauptsächlich in Organen abgelagert, die reich an Fettgewebe sind, wie gehirn und hoden, und daher können einige Benutzer Anzeichen und Symptome einer Vergiftung für bis zu 12-24h aufgrund der langsamen Freisetzung dieser Substanzen durch Adipozyten (SOLOWIJ; PESA, 2008).

THC Halbwertszeit kann von 20h bis 10 bis 13 Tage und die vollständige Eliminierung kann bis zu 30 Tage dauern. Wenn also eine Person Marihuana wiederholt in kleineren Abständen verwendet, erhöht sich die THC-Konzentration im Blut, was zu intensiveren Wirkungen führt (SOLOWIJ; PESA, 2008; CORTEZ, 2009).

Marihuana-Zigaretten, weithin gefunden, obwohl in einigen Ländern wie Brasilien verboten, sind auch als "basiert" bekannt, bestehend aus etwa 0,3-1g Marihuana mit einer Konzentration von 1-15\% Delta-9-THC (2,5 bis $150 \mathrm{mg} \mathrm{THC}$ ). In diesem Zusammenhang wird beobachtet, dass die Produktion von Euphorieeffekten bei einer Mindestkonzentration von 1\% THC oder 1 Zigarette von 2 bis $5 \mathrm{mg}$ auftritt. Und im Gegensatz zu dem, was viele denken, treten die Symptome einer Vergiftung bereits einige Minuten nach gebrauchen auf (RIBEIRO; MARQUES, 2008; SOLOWIJ; PESA, 2008).

In Bezug auf die Auswirkungen von Marihuana, in einigen Studien Symptome im Zusammenhang mit Freizeitgebrauch und Missbrauch dieser Droge berichtet. Auf akademischer Ebene können die Auswirkungen des Marihuanakonsums jedoch in akute und chronische Symptome unterteilt werden. Akute Wirkungen werden als euphorisch eingestuft (erhöhtes sexuelles Verlangen, Gefühl der Verlangsamung der Zeit, erhöhtes Selbstvertrauen und Größe, unmotiviertes Lachen, Heiterkeit, Entspannung und erhöhte Wahrnehmung von Farben, Klängen, Texturen und Geschmack), körperliche Effekte (Tachykardie, Bindehauthyperämie, Trockenmund, Unterkühlung, Schwindel, psychomotorische Retardierung, motorische

RC: 80477

Disponível em:

https://www.nucleodoconhecimento.com.br/gesundheit/marihuanakonsum 
Inkoordination, verminderte Sehschärfe, erhöhte Hörschärfe, Bronchodilatation, erhöhter Appetit, Husten und Mydriasis, Unrealisation, Depression, Halluzination, Illusion, Schläfrigkeit, Konzentrationsstörungen und Kurzzeitgedächtnis, Panikattacken und Paranoia) (SOLOWIJ; PESA, 2008; CORTEZ, 2009).

\subsection{MISSBRAUCH UND KRANKHEIT}

Einige Autoren induzieren, dass der Konsum von psychoaktiven Substanzen durch Zeiten und Kulturen gegangen ist, in religiösen Ritualen verwendet wurde, als therapeutische Methode und auf der Suche nach Vergnügen, wobei hedonismus als Referenz in diesem Prozess betont wird (COUTINHO; ARAÚJO; GONTIÈS, 2004).

Kurzfristig kann man die schädlichen Auswirkungen des Marihuanakonsums, wie z. B. motorische Defizite, bei der Ausführung von Aufgaben wie dem Fahren beobachten; kognitive Nk- und Gedächtnisprobleme und damit Lernschwierigkeiten. Auf der anderen Seite, langfristige Nutzung kann durch chronischen Husten gekennzeichnet werden,veränderte Immunität, reduzierte Testosteronspiegel und die Entwicklung von psychischen Erkrankungen wie Schizophrenie, Depression, Panikattacken, Angst, Reizbarkeit, Demotivation durch leben, Halluzinationen und Depersonalisierung. Je höher die verbrauchte Dosis, desto intensiver werden die Auswirkungen und Raten von Krankheiten sein. Die Abhängigkeit vom Marihuanakonsum erfolgt im Ausmaß seines Konsums. Zu den Nebenwirkungen zählen Amnesie, mangelnde Aufmerksamkeit, motorische Probleme, Die Entstehung von Abhängigkeiten und Entzugskrisen (RIBEIRO; MARQUES, 2008; SOLOWIJ; PESA, 2008).

Einige Studien zeigen kognitive Dysfunktion bei Cannabis konsumenten während der Vergiftung, mehrere Stunden nach dem Akt des Rauchens es, nach ein paar Tagen und anhaltende Schäden für mehr als 1 Monat nach Beendigung der Verwendung. Die Chronik der kognitiven Veränderungen hängt vom Organismus, Dosis und Periodizität, dass das Individuum nutzt das Kraut, aber es ist wichtig zu betonen,

RC: 80477

Disponível em:

https://www.nucleodoconhecimento.com.br/gesundheit/marihuanakonsum 
dass seit der ersten Verwendung des Medikaments und nach dem Beginn der THCWirkung, diese psychischen Verluste bereits manifestiert sind (RIBEIRO; MARQUES, 2008).

Die Beziehungen zwischen kognitiver Leistungsfähigkeit und Häufigkeit des Cannabis konsums können auf eine Restwirkung akuter oder chronischer Vergiftung hindeuten, die sich wahrscheinlich mit der Einstellung des Konsums auflösen würde. Einige Studien berichten, dass die Wiederherstellung der kognitiven Funktion nicht vollständig aufgeklärt ist, aber in einigen Quellen wird festgestellt, dass die Erholung nach 1 Monat Abstinenz auftreten kann oder nicht in 1 Monat auftritt oder Erholung kann nur teilweise sein (RIBEIRO; MARQUES, 2008; SOLOWIJ; PESA, 2008).

Die Verwendung von Marihuana kann auch zu strukturellen Veränderungen im Gehirn führen, wie die Verringerung der Volumen des Hippocampus und Amygdala, und es kann noch nicht bestätigt werden, dass diese Effekte reversibel sind. Aber durch diesen Prozess kann man den wahren Grund verstehen, dass kognitive Beeinträchtigung häufiger im Gedächtnis, Exekutivfunktion, Aufmerksamkeit und hemmungslose Kontrolle erkannt wird (SOLOWIJ; PESA, 2008).

In diesem Zusammenhang ist es interessant festzustellen, dass das Subjekt, das Marihuana verwendet, einen offensichtlichen Grund haben kann oder auch nicht, einen solchen Konsum durchzuführen, und unter den wichtigsten stimulierenden Faktoren für den Konsum der Droge kann erwähnt werden: Flucht aus den Problemen, Neugier, Suche nach Freuden und sozialem Einfluss.

\section{FAZIT}

Es kann beobachtet werden, dass die Verwendung von Marihuana eine Reihe von akuten und chronischen Wirkungen impliziert, sowie Rest, im Zusammenhang mit dem Wirkmechanismus von THC. Darüber hinaus haben Benutzer dieses Medikaments oft Probleme, die ihre Familie, berufliche und soziale Leben betreffen.

RC: 80477

Disponível em:

https://www.nucleodoconhecimento.com.br/gesundheit/marihuanakonsum 
In diesem Zusammenhang ist es interessant zu beobachten, dass das Subjekt, das Marihuana verwendet, einen offensichtlichen Grund haben kann oder auch nicht, um den Gebrauch durchzuführen, aber was den Wunsch weckt, Studien auf der Brücke zwischen Vergnügen und Krankheit zu fördern, ist das, was hinter der Suche nach der Substanz steckt, eine Tatsache, die außerhalb der Augen des Benutzers und des Bewussten liegt.

In der vorliegenden Studie, einige Beweise, die die Gründe erklären können, warum Einzelpersonen entscheiden, Marihuana zu verwenden. Meistens sind die akuten Auswirkungen von Euphorie, Vergnügen und Entspannung gewünscht, im Gegensatz dazu wird beobachtet, dass die Schäden im Zusammenhang mit diesem Gebrauch am Ende die illusorischen Vorteile, die das Medikament bietet, zu überwinden, so dass Freude zu einer Krankheit wird.

\section{VERWEISE}

CORTEZ, P. Possíveis efeitos cognitivos e psicomotores em usuários crônicos de Cannabis. 2009.

COUTINHO, M. P. L.; ARAÚJO, L. F.; GONTIÈS, B. Uso da maconha e suas representações sociais: estudo comparativo entre universitários. Psicologia em Estudo, Maringá, v. 9, n. 3, p. 469-477, set./dez. 2004.

FERNANDES, T. F.; MONTEIRO, B. M. M.; SILVA, J. B. M.; OLIVEIRA, K. M.; VIANA, N. A. O.; GAMA, C. A. P.; GUIMARÃES, D. A. Uso de substâncias psicoativas entre universitários brasileiros: perfil epidemiológico, contextos de uso e limitações metodológicas dos estudos. Cad. Saúde Colet., 2017, Rio de Janeiro, 25 (4): 498-507.

RC: 80477

Disponível em: 
FERRARI, C. R. Cannabis. Universidade de São Paulo. Instituto de Física de São Carlos - Psicologia da Educação - SLC 0631. Licenciatura em Ciências Exatas. 2016. 15p.

GARCIA, F. D. Manual de abordagem de dependências químicas. ED. Utopika, 2014, 386p.

RIBEIRO, M; MARQUES, A.C. P. R. Projeto diretrizes: Abuso e dependência Maconha. 2008.

ROSA, P.; ROSA, M. G. Políticas sobre cannabis: um estudo comparativo sobre os modelos da Espanha. Geographia Opportuno Tempore Universidade Estadual de Londrina EISSN: 2358-1972, Volume 4, Número 1, 2018 Uruguai e Colorado/EUA, 2018.

SOLOWIJ, N,; PESA, N. Anormalidades cognitivas no uso da cannabis. 2010.

Eingereicht: März, 2021.

Genehmigt: März, 2021.

RC: 80477

Disponível em: 